\title{
ASCA Observations of Blazars and Multiband Analysis
}

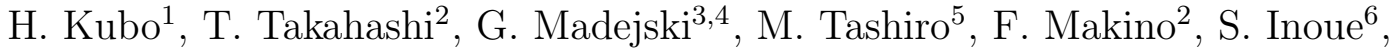 \\ and F. Takahara ${ }^{7}$
}

\begin{abstract}
We present data for 18 blazars observed with the $\mathrm{X}$-ray satellite $A S C A$, half of which were also observed contemporaneously with the EGRET instrument onboard Compton Gamma-ray Observatory as parts of multi-wavelength campaigns. The observations show a clear difference in the spectra between three subclasses of blazars, namely the High-energy peaked BL Lac objects (HBLs), Low-energy peaked BL Lac objects (LBLs), and quasar-hosted blazars (QHBs). The ASCA X-ray spectra of HBLs are the softest, with the power law energy index $\alpha \sim 1-2$, and they form the highest observable energy tail of the low energy (LE, synchrotron) component. The X-ray spectra of the QHBs are the hardest $(\alpha \sim 0.6)$ and are consistent with the lowest observable energy end of the high energy (HE, Compton) component. For LBLs, the X-ray spectra are intermediate. We find that the radiation process responsible for the HE peak for HBLs can be explained solely by Doppler-boosted Synchrotron-Self-Compton (SSC) emission, with the Doppler factor $\delta$ consistent with the VLBI and/or $\gamma$-ray variability data. For many QHBs, on the other hand, the $\gamma$-rays cannot be solely due to the SSC mechanism unless $\delta$ is significantly in excess of that inferred from VLBI data. We consider an alternative scenario consistent with the measured values of $\delta$, where the SSC component is still present in QHBs and it dominates in the $\mathrm{X}$-ray band, but it is below the observed $\gamma$-ray spectrum.
\end{abstract}

\footnotetext{
${ }^{1}$ The Institute of Physical and Chemical Research (RIKEN), 2-1 Hirosawa, Wako, Saitama, 351-0198, Japan

${ }^{2}$ Institute of Space and Astronautical Science, Sagamihara, Kanagawa, 229-8510, Japan

${ }^{3}$ Laboratory for High Energy Astrophysics, Code 662, NASA GSFC, MD 20771, USA

${ }^{4}$ University of Maryland, College Park, MD 20742, USA

${ }^{5}$ Department of Physics, University of Tokyo, Bunkyo-ku, Tokyo, 113-0033, Japan

${ }^{6}$ Tokyo Metropolitan University, 1-1 Minami Osawa, Hachioji, 192-0397, Japan

${ }^{7}$ Department of Earth and Space Science, Graduate school of Science, Osaka University, 1-1 Machikaneyama, Toyonaka, Osaka, 560-0043, Japan
} 
With an assumption that the peak of the SSC emission is on the extrapolation of the X-ray spectrum, and adopting $\delta$ of 10 , we infer the magnetic field $B$ to be $0.1-1$ Gauss, and Lorentz factors $\gamma_{b}$ of electrons radiating at the peak of the $\nu F(\nu)$ spectrum of $\sim 10^{3}$ for QHBs; this is much lower than $\gamma_{b} \sim 10^{5}$ for HBLs, even though the values of $B$ are comparable in the two sub-classes. This difference of $\gamma_{b}$ is most likely due to the large photon density expected in QHBs (e.g. from thermal components visible in these objects) as compared with that of HBLs; Compton upscattering of these photons may well provide the observed $\mathrm{GeV}$ flux.

Subject headings: BL Lacertae objects: general — quasars: general — radiation mechanisms: non-thermal - X-rays: galaxies

\section{Introduction}

The overall electromagnetic spectra of blazars - a class of active galactic nuclei (AGNs) that includes BL Lac objects and Optically Violently Variable (OVV) quasars are believed to be dominated by Doppler-boosted radiation from relativistic jets pointing closely to our line of sight (Blandford \& Rees 1978; Blandford \& Königl 1979; Urry \& Padovani 1995 for a review of radio loud AGNs). The VLBI studies of these objects show compact radio cores on milli-arcsecond angular scale with jet-like structures which often

show superluminal motion, with apparent speeds $v / c \sim 5-10$ (e.g., Vermeulen \& Cohen 1994). Apparent variability time scale and luminosity amplification depend on various powers of the "beaming" (Doppler) factor $\delta$ (e.g., Lind \& Blandford 1985), defined via $\delta=\Gamma_{j}^{-1}(1-\beta \cos \theta)^{-1}$, where $\Gamma_{j}$ is the bulk Lorentz factor of the emitting matter, $\beta=v / c$, and $\theta$ is the angle of motion with respect to the line of sight.

Blazars are commonly detected as $\gamma$-ray sources. The EGRET instrument onboard the Compton Gamma-Ray Observatory (CGRO) has so far detected emission in the GeV range from $\sim 50$ blazars (Fichtel et al. 1994; Thompson et al. 1995; Mattox et al. 1997; Mukherjee et al. 1997); $\gamma$-ray emission has been detected up to the TeV range from the nearby BL Lac objects Mkn 421 and Mkn 501 with ground-based Cherenkov telescopes (Punch et al. 1992; Petry et al. 1993; Quinn et al. 1996; Bradbury et al. 1997). As these sources show large luminosity and compact emission regions in the spectral range where the opacity to pair production via $\gamma \gamma \rightarrow e^{+} e^{-}$is large, it is generally accepted that the $\gamma$-ray emission is anisotropic and Doppler-boosted as well (Maraschi, Ghisellini \& Cellotti 1992; Mattox et al. 1993; Dondi \& Ghisellini 1995; Buckley et al. 1996), suggesting that 
the entire observed electromagnetic emission arises in the jet. As these objects emit in practically every observable waveband, any study of the structure and physical conditions in the jets requires broad-band spectral observations, which, given the rapid large amplitude flux variability, must be conducted simultaneously.

The overall spectra of blazars have two pronounced components: one peaking at low energies (LE), $10^{13}-10^{17} \mathrm{~Hz}$ (e.g., Sambruna, Maraschi, \& Urry 1996), and another peaking at high energies (HE), in the $\gamma$-rays (e.g., von Montigny et al. 1995). For the blazars that are hosted in quasars (QHBs), and for BL Lac objects discovered via radio-selection techniques (the so-called "Low-energy peaked BL Lacs" or LBLs), the LE component peaks in the infrared. For the the majority of BL Lac objects - those found as a result of their $\mathrm{X}$-ray emission - it peaks in the ultraviolet or even in the soft X-rays (Giommi, Ansari \& Micol 1995; Sambruna, Maraschi, \& Urry 1996; Padovani \& Giommi 1996; Fossati et al. 1997a), and thus they are named "High-energy peaked BL Lacs (HBLs)" (Padovani \& Giommi 1995). The local power-law shape, the smooth connection of the entire radio - to - UV (and, for the HBLs, soft X-ray) continuum, as well as the relatively high level of polarization observed from radio to the UV, imply that the emission from the LE component is most likely produced via the synchrotron process of relativistic particles radiating in magnetic field. This view is strongly supported by spectral variability observed in X-rays in a number of HBLs, where the variability at lower X-ray energies lags behind the more energetic X-rays (Tashiro 1992; Sembay et al. 1993; Kohmura et al. 1994; Tashiro et al. 1995; Takahashi et al. 1996)

The HE component, on the other hand, peaks in the $\gamma$-ray band, in the MeV - to $\mathrm{GeV}$ range, and, in the case of a few HBLs, it extends to the TeV range; it is believed to be produced via Comptonization by the same particles that radiate the LE component. The source of the "seed" photons, can either be the synchrotron radiation, internal to the jet - as in the Synchrotron-Self-Compton (SSC) models (Rees 1967; Jones, O'Dell \& Stein 1974; Königl 1981; Ghisellini, Maraschi \& Treves 1985; Band \& Grindlay 1985; Ghisellini \& Maraschi 1989; Maraschi, Ghisellini \& Cellotti 1992; Bloom \& Marscher 1996; Mastichiadis $\&$ Kirk 1997). Alternatively, these can be external to the jet, as in the External Radiation Compton (ERC) models: either the UV accretion disk photons (Dermer, Schlickheiser, \& Mastichiadis 1992; Dermer, Sturner, \& Schlickeiser 1997), or these UV photons reprocessed by the emission line clouds and/or intercloud medium (Sikora, Begelman \& Rees 1994; Blandford \& Levinson 1995), or else, IR radiation ambient to the host galaxy (Sikora, Begelman \& Rees 1994). The ratio of the power in the HE to the LE components is systematically larger for QHBs than for BL Lac objects (Maraschi, Ghisellini \& Celotti 1994a; Dondi \& Ghisellini 1995; Sambruna 1997a; Urich, Maraschi \& Urry 1997; Fossati et al. 1997b). 
If we assume that the LE component is due to the synchrotron radiation, its peak frequency is determined by the intensity of magnetic field and the distribution function of electron energies, while the location of the HE peak is related to the distribution functions of electron and target photon energies. The ratio of the luminosity of these components $\left(L_{H E} / L_{L E}\right)$, in the context of this synchrotron plus Compton model, is expected to reflect the ratio of energy densities of photon and magnetic fields in the jet.

This paper reports the $\mathrm{X}$-ray spectra of 18 blazars measured by $A S C A$ in the context of their multi-band emission. The ASCA observations and results are described in $\S 2$, followed by multi-band analysis and discussion in $\S 3$. Summary of this paper is presented in $\S 4$. Throughout this paper we use $H_{0}=75 \mathrm{~km} \mathrm{~s}^{-1} \mathrm{Mpc}^{-1}, q_{0}=0.5$.

\section{ASCA observations and multiband results}

X-ray satellite ASCA (Tanaka, Inoue \& Holt 1994) observed 18 blazars (cf. Table 1), half of which were also observed contemporaneously with EGRET as parts of multiband campaigns; such simultaneous observing campaigns are essential for these highly variable objects.

We applied the standard screening criteria to the data. The minimum cutoff rigidity is $6 \mathrm{GeV} / \mathrm{c}$. The minimum elevation angle is 10 and 5 degree respectively for the Solid-state Imaging Spectrometer (hereafter SIS; Burke et al. 1991; Yamashita et al. 1997) and the Gas Imaging Spectrometer (GIS; Ohashi et al. 1996; Makishima et al. 1996). The minimum angle between the target and the illuminated Earth's limb is 20 degree for the SIS. We rejected the data obtained during the passages through the South Atlantic Anomaly. We extract the source counts from a region of a radii of 3 arcmin and 4-6 arcmin respectively. For the GIS we chose a radius in proportion to the brightness of a source as long as the ratio of signal to background is one. The background counts were taken from a source-free region in the same image as the target for a source whose source count rate is below 0.2 $\operatorname{cts~s}^{-1}$. For sources whose count rate is above $0.2 \operatorname{cts~s}^{-1}$, there is no image region where contamination from the source is negligible. For those cases, we took background from the same detector region as the location of the source, but from blank sky observations.

We fitted the spectra using the detector response v1.1 $\alpha$ for SIS and v4.0 for GIS, by a power law with absorption at low energies (cross section from Morrison \& McCammon 1983). In most cases, the data were well-described by this model, where the absorption was consistent with the Galactic value, although for HBLs the spectra are convex and this model is too simple (Sambruna et al. 1994; Tashiro et al. 1995; Takahashi et al. 1996; 
Sambruna et al. 1997b; Kubo 1997). But in all cases, a power law model with Galactic absorption suffices in the $2-10 \mathrm{keV}$ range. When there is more than one ASCA observation during one viewing period of EGRET, we used an index determined from the summed data over the period of contemporaneous observations with EGRET.

Figure 1 shows the distribution of the $2-10 \mathrm{keV}$ energy indices of the blazars obtained with ASCA. This figure clearly shows that the X-ray spectra of HBLs are the softest, with the power law energy index $\alpha \sim 1-2$, and they form the highest observable energy tail of the LE component. X-ray spectra of the QHBs are the hardest $(\alpha \sim 0.6)$ and are consistent with the lowest observable energy end ("onset") of the HE component. For LBLs, the X-ray spectra are intermediate; in one case, 0716+714, the ASCA spectrum shows hardening with an increasing energy (Fig. 2), similar to the spectrum observed with ROSAT (Cappi et al. 1994). The differences in the spectra between three subclasses were also observed with ROSAT in the soft X-ray band (Sambruna, Maraschi, \& Urry 1996; Urry et al. 1996; Sambruna 1997a; Padovani, Giommi \& Fiore 1997; Comastri et al. 1997). The time variability in ASCA observations is reported in Kubo (1997). It is worthwhile to note that while the energy index for HBLs is often variable on a short time scale, for QHBs the index remains almost constant when the flux changes.

We constructed the multiband spectral energy distribution ranging from radio to $\gamma$-ray band for 18 sources observed with ASCA using above data, published results, the database and the data taken by private communication. The $K$-corrected multiband spectra are shown in Fig. 2. For the $K$-correction, the flux densities are multiplied by $(1+z)^{\alpha-1}$, where $\alpha$ is the energy spectral index at frequency $\nu$, defined such that flux $F(\nu) \propto \nu^{-\alpha}$. We used individual spectral indices when available, otherwise average indices for each subclass of blazars (Sambruna, Maraschi, \& Urry 1996). We plot the simultaneous data when available, and for non-simultaneous data we averaged the flux densities at a given frequency. In order to determine the peak frequency and the luminosity of both the LE and HE components, we performed a empirical third-order polynomial fit to the spectra in $\nu F(\nu)$ (Comastri, Molendi \& Ghisellini 1995). The results are listed in Table 2. Figures 4a and $4 \mathrm{~b}$ show that, for QHBs, the peak frequency of the LE component is lower, $L(L E)$ and the $L(H E) / L(L E)$ ratio is greater than that measured in HBLs. For LBLs, these properties are, again, intermediate.

\section{Discussion}

As we mentioned previously, the two leading models of the high energy emission in blazars invoke Comptonization, of internal (SSC) or external (ERC) seed photons. In 
the following analysis, we assume that both $\mathrm{SSC}$ and $\mathrm{ERC}$ processes may operate in blazars. We then estimate the contribution of the SSC emission in the HE component. In order to calculate the predicted luminosity due to the SSC emission, we assume a simple homogeneous model, in which photons are produced in a region of radius $R$ and with a constant magnetic field $B$.

We considered the radiation by a single population of relativistic electrons, with a broken power law distribution of Lorentz factors $\gamma_{e l}$, and a break point at $\gamma_{b}$ (similar to e.g. Sambruna, Maraschi, \& Urry 1996). We also assume that the radiation spectrum of the LE component peaks at a frequency corresponding to that radiated by the electrons with $\gamma_{b}$.

The peak frequency of the synchrotron component in the observer frame, $\nu_{\text {sync }}$, is then given as, when pitch angle is $\pi / 2$ :

$$
\nu_{\text {sync }}=1.2 \times 10^{6} \gamma_{b}^{2} B \frac{\delta}{(1+z)} \quad \mathrm{Hz}
$$

where $\mathrm{B}$ is in Gauss. If the electron energy is still in the Thomson regime, $\left(\gamma_{e l} \times h \nu_{\text {sync }}<<m_{e} c^{2}\right)$, the expected peak of the SSC component in the observer frame $\left(\nu_{S S C}\right)$ is $\nu_{S S C}=4 \gamma_{b}^{2} \nu_{\text {sync }} / 3$. The ratio of the observed luminosity of the SSC component $L_{S S C}$ to the observed synchrotron luminosity $L_{\text {sync }}$ is:

$$
\frac{L_{S S C}}{L_{\text {sync }}}=\frac{u_{\text {sync }}}{u_{B}}
$$

where the $u_{\text {sync }}=L_{\text {sync }} /\left(4 \pi R^{2} c \delta^{4}\right)$ is the rest-frame energy density of the synchrotron photons, and $u_{B}=B^{2} /(8 \pi)$ is the magnetic field energy density.

To check the validity of the assumption that the observed HE component is solely due to the SSC emission, we calculated the beaming factor $(\delta)$, which is given from above equations:

$$
\delta^{2}=1.6 \times 10^{12} \frac{L_{\text {sync }}}{c R^{2}}\left(\frac{L_{\text {sync }}}{L_{S S C}}\right) \frac{\nu_{S S C}^{2}}{\nu_{\text {sync }}^{4}} \frac{1}{(1+z)^{2}}
$$

where $L$ is in erg $\mathrm{s}^{-1}, \nu$ in $\mathrm{Hz}, c$ in $\mathrm{cm} \mathrm{s}^{-1}$, and $R$ in $\mathrm{cm}$. We estimate $R$ from the shortest observed variability (doubling) time scale $\Delta t$ observed in any wavelength, as given in Table 2. Assuming that $R \lesssim c \delta \Delta t /(1+z)$, then Eq. 3 can be rewritten as:

$$
\delta^{4} \gtrsim 1.6 \times 10^{12} \frac{L_{\text {sync }}}{c^{3} \Delta t^{2}}\left(\frac{L_{\text {sync }}}{L_{S S C}}\right) \frac{\nu_{S S C}^{2}}{\nu_{\text {sync }}^{4}}
$$

where $\Delta t$ is in s, and other quantities are as in Eq. 3. An application of this equation to the data in Table 2 assuming $L_{s y n c}=L_{L E}, L_{S S C}=L_{H E}, \nu_{s y n c}=\nu_{L E}, \nu_{S S C}=\nu_{H E}$ 
implies that the lower limits of $\delta$ for HBLs are $\sim 3$ or less, which is consistent with the VLBI results (cf. Vermeulen \& Cohen 1994), and the limits obtained from the arguments of the $\gamma$-ray opacity (cf. Dondi \& Ghisellini 1995). However, for 4 QHBs, where the $\gamma$-ray flux severely dominates the radiative output, we derive values of $\delta$ that are much larger than the VLBI results (see Table 2). This suggests that an additional emission mechanism - such as the ERC process - may contribute significantly in the $\gamma$-ray regime, dominating over the SSC flux, and the values of $\nu_{S S C}$ and $L_{S S C}$ are very different than $\nu_{H E}$ and $L_{H E}$, with the SSC component "hidden" well below the ERC component.

However, the fact that the QHBs have X-ray spectra which are hard, with $\alpha \sim 0.6$, and which are not located on the extrapolation of the synchrotron optical / UV spectra, implies that the $\mathrm{X}$-rays observed in QHBs are due to a separate emission process than synchrotron. The fact that for most of QHBs the $\gamma$-ray spectra are above the extrapolation of X-ray spectra (Fig. 2) suggests that the dominant process is different for $\mathrm{X}$-rays than it is for $\gamma$-rays. One explanation is that the SSC process dominates in the $\mathrm{X}$-ray range, while the ERC process dominates in $\gamma$-rays (Inoue \& Takahara 1996). With the assumption that $\mathrm{SSC}$ process is dominant in $\mathrm{X}$-rays for QHBs, we estimate the location of the $\left(\nu_{S S C}, L_{S S C}\right)$ point in the $\log (\nu)-\log (\nu F(\nu))$ space by the following method. We assume that it lays on or below the extrapolation of the ASCA spectrum (line (a) in Fig. 3), but above the highest value of $\nu F(\nu)$ measured by $A S C A$. Since the spectra of QHBs generally have $\alpha<1$ and thus $\nu F(\nu)$ is the highest at the end of the $A S C A$ bandpass $\left(10 \mathrm{keV} \simeq 2 \times 10^{18} \mathrm{~Hz}\right)$, this second limit is equivalent to $\nu_{S S C} L_{S S C}>2 \times 10^{18} \mathrm{~Hz} L_{10 \mathrm{keV}}$ (line (b) in Fig. 3). We further constrain $L_{S S C}$ using Eq. 3; once we assume a given $\delta$, there is a unique relationship between $L_{S S C}$ and $\nu_{S S C}$ described as:

$$
L_{S S C}=1.6 \times 10^{12}\left(\frac{L_{\text {sync }}^{2}}{c R^{2} \nu_{\text {sync }}^{4} \delta^{2}(1+z)^{2}}\right) \nu_{S S C}^{2} \quad \operatorname{erg~s}^{-1}
$$

where $L, R, c$, and $\nu$ are in the same units as in Eqs $3 \& 4$. The VLBI data and $\gamma$-ray opacity argument suggest that $5<\delta<20$ for most blazars (e.g. Vermeulen \& Cohen 1994; Dondi \& Ghisellini 1995). This corresponds to the lines (c) and (d) in Fig. 3, respectively for $\delta=5$ and 20. The above four constraints correspond to the shaded area of Fig. 3, where for illustration, we use the overall spectral energy distribution for the QHB CTA 102.

Since we have to use a unique value in calculating the physical parameters, we use $\delta=$ 10 as a geometrical mean between 5 and 20. The $L_{S S C}-\nu_{S S C}$ line calculated from Eq. 5 corresponding to $\delta=10$ intersects both the extrapolation of the ASCA spectrum and the highest $A S C A$ value, and the intersections yield the lower and upper values for both $L_{S S C}$ and $\nu_{S S C}$. We adopt a mean of these values, which are given in Table 2, and plotted in Figure 4c. We used Ginga data instead of ASCA data for 3C 279 because a simultaneous 
campaign from radio to $\gamma$-ray bands was conducted during Ginga observation. The values for the other blazars where $\delta$ derived from Eq. 7 is $<20$, are calculated by assuming $L_{S S C}$ $=L_{H E}$. For LBL AO0235+164 where $\delta$ derived from Eq. 4 is $>20$, we assume $L_{S S C}=$ $L_{L E}$ because the $A S C A$ spectra of AO0235+164 is thought to be mixture of the LE and HE component, as discussed by Madejski et al. (1996) based on the ROSAT and ASCA spectra, so that the above method may be inappropriate. For two QHBs (3C 273, PKS 0208-512) the lower limits of $\delta$ are $\sim 5$. The fact that observed $\gamma$-ray flux of PKS 0208-512 is much higher than the extrapolation of $A S C A$ spectrum implies the $\gamma$-ray peak is not solely due to SSC mechanism. Therefore we applied the above method to this source. On the other hand, since the $\gamma$-ray spectrum of 3C 273 is below the extrapolation of ASCA spectrum, the $\gamma$-ray emission is assumed to be due to SSC mechanism so there, we assume $L_{S S C}=$ $L_{H E}$. It is important to note, however, that $3 \mathrm{C} 273$ is unique as compared to other blazars considered here in that the "blue bump" is very pronounced, and thus it is unlikely that the jet dominates the entire electromagnetic emission, and therefore, a more complex analysis is necessary (see, e.g., von Montigny et al. 1997 for further discussion).

Once we obtain $L_{S S C}$, and $\nu_{S S C}$, we can calculate the strength of the magnetic field and the electron Lorentz factor $\gamma_{b}$ from Eq. (1), (2) and those are given as follows:

$$
\begin{gathered}
B=0.27\left(\frac{R_{\mathrm{pc}}}{10^{-2}}\right)^{-1}\left(\frac{\delta}{10}\right)^{-2} \sqrt{\left(\frac{L_{\text {sync }}}{10^{46}}\right)\left(\frac{L_{\text {sync }}}{L_{S S C}}\right)} \text { Gauss } \\
\gamma_{b}=1.8 \times 10^{3}\left(\frac{R_{\mathrm{pc}}}{10^{-2}}\right)^{1 / 2}\left(\frac{\delta}{10}\right)^{1 / 2}\left(\frac{\nu_{\text {sync }}(1+z)}{10^{13}}\right)^{1 / 2}\left[\left(\frac{L_{\text {sync }}}{10^{46}}\right)\left(\frac{L_{\text {sync }}}{L_{\text {ssc }}}\right)\right]^{-1 / 4}
\end{gathered}
$$

where $R_{\mathrm{pc}}$ is size of emission region in parsecs, and other quantities are as in Eqs 3, 4, \& 5 . As before, the upper limit of the size $R$ can be estimated from the observed time variability $(\Delta t)$ from Table 2 , given by $R \lesssim c \Delta t \delta /(1+z)$.

Our calculated values of $B$ and $\gamma_{b}$ are plotted respectively in Figures $4 \mathrm{~d}$ and $4 \mathrm{e}$. In these Figures, we also plot the values calculated with $R=0.01 \mathrm{pc}$, which would correspond to an observed variability time scale of $\sim 1$ day.

From our analysis, the magnetic field for blazars observed with ASCA is inferred to be 0.1 - 1 Gauss. The value of $B$ is comparable between the different subclasses of blazars, although $B$ is somewhat lower in HBLs than in QHBs. With these values of $B$, we estimate $\gamma_{b}$ to be $10^{3}-10^{4}$ for QHBs, and $10^{5}$ for HBLs. The differences of $\gamma_{b}$ between different sub-classes of blazars imply that the relativistic electrons are accelerated to higher energies in HBLs than in QHBs. Alternatively, higher $\gamma_{b}$ in HBLs might be obtained by increasing $\delta$. However, in those objects, we believe that there is no contribution to the $\gamma$-ray production from other mechanisms besides SSC, and thus the observed $L_{H E}$ is $L_{S S C}$. In such case, $\gamma_{b}$ 
depends on $\delta$ only linearily (cf. Eq. 7 and $R \lesssim c \Delta t \delta /(1+z)$ ), and thus varying $\delta$ to be 5 or 20 respectively decreases or increases our derived $\gamma_{b}$ only by a factor of two, which is small when compared to the large difference of $\gamma_{b}$ calculated by us (cf. Fig. 4e).

In QHBs the strong optical and UV line emission implies a presence of dense external radiation fields. This means that in the frame of reference of the jet, these can easily dominate over the internal synchrotron radiation, resulting in the ERC emission dominating over the SSC emission in $\gamma$-ray band (e.g., Sikora et al. 1997). It is likely that the difference of $\gamma_{b}$ is most likely due to the large photon density in QHBs as compared with that of HBLs. It should be noted that $\mathrm{TeV}$ photons have been observed only from HBLs, where we calculate higher values of $\gamma_{b}$.

\section{Summary}

The non-thermal emission from blazars, observed from radio to $\mathrm{GeV} / \mathrm{TeV} \gamma$-rays, is thought to be the result of radiation of very energetic particles via both synchrotron and Compton processes. The overall spectra of all blazars over this wide range of energies appear similar from one object to another, forming two distinct peaks in the $\nu F(\nu)$ representation. The $\mathrm{X}$-ray regime is important, as it is where the emission due to both processes overlaps: for the High-energy peaked BL Lac objects (HBLs), X-rays form the high energy tail of the synchrotron emission, while for blazars showing quasar-like emission lines (QHBs), they form the lowest observable energy end of the Comptonized spectrum. The X-ray spectra of Low-energy peaked BL Lac objects (LBLs) appear intermediate, which may depend on the level of activity of the object. An application of the synchrotron self-Compton (SSC) model to the overall spectral distribution and variability data of 18 blazars observed by ASCA implies that for the HBLs, the SSC model can explain all available data quite well, and implies relativistic Doppler factors $\delta$ in the range of $5-20$, consistent with the those derived from the VLBI data and from the limits inferred from $\gamma$-ray opacity to pair production, $\gamma \gamma \rightarrow e^{+} e^{-}$. Further support for it comes from the good agreement of values physical parameters of the radiating plasma as inferred from the energetics requirement to produce the $\mathrm{TeV} \gamma$-rays, with that inferred from the spectral variability observed in $\mathrm{X}$-rays in a number of objects.

The situation in QHBs (and in some LBLs) is quite different, as the application of the SSC model implies the values of $\delta$ much in excess of values inferred from the $\gamma$-ray opacity arguments, or the VLBI data. This discrepancy can be eliminated if we adopt a scenario where the observed $\mathrm{GeV} \gamma$-rays are produced by Comptonization of external photons (via the ERC process), while the flux produced by the SSC process - while present and possibly 
responsible for the $\mathrm{X}$-ray emission - in the $\mathrm{GeV}$ range is well below the ERC emission. With this we estimated the $L_{S S C}$ of QHBs, by assuming $\mathrm{X}$-ray emission in $A S C A$ band is due to SSC process.

The inferred magnetic fields in all blazars is comparable $-\sim 0.1$ to 1 Gauss - but, what distinguishes QHBs and HBLs is the much lower value of Lorentz factors of electrons radiating at the peak, $\sim 10^{3}-10^{4}$ for QHBs against $\sim 10^{5}$ for HBLs. However, the details of the jet structure, and in particular, the question of the particle acceleration, still remain open, with the hope that the future missions - such as the upcoming satellites ASTRO-E and GLAST - will further advance our knowledge of the details of physical processes in these extreme, enigmatic objects.

This research has made use of NASA/IPAC Extragalactic Database (NED) and data from the University of Michigan Radio Astronomy Observatory (UMRAO). We thank H. Teräsranta and M. Tornikoski for allowing us to use their unpublished radio data; M. Sikora for many useful discussions and comments; collaborators of ASCA observation, W. Collmar, J. G. Stacy, S. Sembay, A. Yamashita, E. Idesawa, and J. Kataoka; and the referee, C. M. Urry, for valuable comments. G. M acknowledges the support of NASA grant NAG5-4106 and of the Grants-in Aid for Scientific Research by Ministry of Education, Culture, and Science (Monbusho) of Japan (08044103). 


\section{REFERENCES}

Aharonian, F., et al. 1997, A\&A, 327, L5

Band, D. L. \& Grindlay, J. E. 1985, ApJ, 298, 128

Bersanelli, M., et al. 1992, AJ, 104, 28

Bertsch, D. L., et al. 1993, ApJ, 405, L21

Blandford, R. D., \& Rees, M. J. 1978, in Pittsburgh Conference on BL Lac Objects, ed. A. N. Wolfe (Pittsburgh: University of Pittsburgh), p. 328

Blandford, R. D., \& Königl, A. 1979, ApJ, 232, 34

Blandford, R. D., \& Levinson, A. 1995, ApJ, 441, 79

Bloom, S. D., et al. 1994, AJ, 108, 398

Bloom, S. D. \& Marscher, A. P. 1996, ApJ, 461, 657

Blom, J. J., et al. 1995, A\&A, 298, L33

Bradbury, S. M., et al. 1997, A\&A, 320, L5

Buckley, J. H., et al. 1996, ApJ, 472, L9

Burke, B. E., et al. 1991, IEEE Trans. ED-38, 1069

Cappi, M., et al. 1994, MNRAS, 271, 438

Collmar, W., et al. 1998, in preparation

Comastri, A., Molendi, S., \& Ghisellini, G. 1995, MNRAS, 277, 297

Comastri, A., et al. 1997, ApJ, 480, 534

Courvoisier, T. J. L., et al. 1988, Nature, 335, 330

Dermer, C. D., Schlickeiser, R., \& Mastichiadis, A. 1992, A\&A, 256, L27

Dermer, C. D., Sturner, S. J., \& Schlickeiser, R. 1997, ApJS, 109, 103

Dickey, J. M. \& Lockman, F. J. 1990, ARA\&A, 28, 215

Dondi, L., \& Ghisellini, G. 1995, MNRAS, 273, 583 
Elvis, M., Wilkes, B. J., \& Lockman, F. J. 1989, AJ, 97, 777

Falomo, R., et al. 1993, ApJ, 402, 532

Falomo, R., Scarpa, R., \& Bersanelli, M. 1994, ApJS, 93, 125

Feigelson, E. D., et al. 1986, ApJ, 302, 337

Fichtel, C. E., et al. 1994, ApJS, 94, 551

Fossati, G. et al. 1997a, MNRAS, 289, 136

Fossati, G., et al. 1997b, in Cracow Conference on Relativistic Jets in AGNs, ed. M. Ostrowski, M. Sikora, G. Madejski, \& M. Begelman, p. 245

Ghisellini, G., Maraschi, L., \& Treves, A. 1985, A\&A, 146, 204

Ghisellini, G., \& Maraschi, L. 1989, ApJ, 340, 181

Giommi, P., Ansari, S. G., \& Micol, A. 1995, A\&AS, 109, 267

Hartman, R. C., et al. 1996, ApJ, 461, 698

Hunter, S. D., et al. 1993a, A\&A, 272, 59

Hunter, S. D., et al. 1993b, ApJ, 409, 134

Idesawa, E., et al. 1997, PASJ, 49, 631

Impey, C. D., \& Neugebauer, G. 1988, AJ, 95, 307

Inoue, S., \& Takahara, F. 1996, ApJ, 463, 555

Jannuzi, B. T., et al. 1993, ApJS, 85, 265

Jones, T. W., O’Dell, S. L., \& Stein, W. A. 1974, ApJ, 188, 353

Kataoka, J., et al. 1998, in preparation.

Kniffen, D. A., et al. 1993, ApJ, 411, 133

Kohmura, Y, et al. 1994, PASJ, 46, 131

Königl, A. 1981, ApJ, 243, 700

Kubo, H. 1997, Ph.D.Thesis, University of Tokyo 
Landau, R., et al. 1983, ApJ, 268, 68

Landau, R., et al. 1986, ApJ, 308, 78

Lin, Y. C., et al. 1995, ApJ, 442, 96

Lind, K. R., \& Blandford, R. D. 1985, ApJ, 295, 358

Macomb, D. J. et al. 1995, ApJ, 449, L99

Madejski, G. et al. 1996, ApJ, 459, 156

Makishima, K., et al. 1996, PASJ, 48, 171

Maraschi, L., Ghisellini, G., \& Cellotti, A. 1992, ApJ, 397, L5

Maraschi, L., Ghisellini, G., \& Celotti, A. 1994a, in IAU Symp. 159, p.221

Maraschi, L., et al. 1994b, ApJ, 435, L91

Mastichiadis, A. \& Kirk, J. G. 1997, A\&A, 320, 19

Mattox, J. R., et al. 1993, ApJ, 410, 609

Mattox, J. R., et al. 1997, ApJ, 481, 95

McHardy, I. M. M., et al. 1992, MNRAS, 256, 655

McNaron-Brown, K., et al. 1995, ApJ, 451, 575

Morrison, R., \& McCammon, D. 1983, ApJ, 270, 119

Mukherjee, R., et al. 1997, ApJ, 490, 116

Neugebauer, G., et al. 1986, ApJ, 308, 815

Nolan, P. L., et al. 1993, ApJ, 414, 82

Nolan, P. L., et al. 1996, ApJ, 459, 100

Ohashi, T., et al. 1996, PASJ, 48, 157

Padovani, P. 1992, MNRAS, 257, 404

Padovani, P. \& Giommi, P. 1995, ApJ, 444, 567

Padovani, P. \& Giommi, P. 1996, MNRAS, 279, 526 
Padovani, P., Giommi, P., \& Fiore, F. 1997, MNRAS, 284, 569

Petry, D., et al. 1993, A\&A, 311, L13

Pian, E., \& Treves, A. 1993, ApJ, 416, 130

Pica, A. J., et al. 1988, AJ, 96, 1215

Punch, M., et al. 1992, Nature, 358, 477

Quinn, J., et al. 1996, ApJ, 456, L83

Rees, M. J. 1967, MNRAS, 137, 429

Remillard, R. A., et al. 1989, ApJ, 345, 140

Rieke, G. H., et al. 1982, ApJ, 263, 73

Sambruna, R. M., et al. 1994, ApJS, 95, 371

Sambruna, R. M., Maraschi, L., \& Urry, C. M. 1996, ApJ, 463, 444

Sambruna, R. M. 1997a, ApJ, 487, 536

Sambruna, R. M., et al. 1997b, ApJ, 483, 774

Sembay, S. et al. 1993, ApJ, 404, 112

Shrader, C. R., Hartman, R. C., \& Webb, J. R. 1996, A\&AS, 120, 599

Sikora, M., Begelman, M. C., \& Rees, M. J. 1994, ApJ, 421, 153

Sikora, M., Madejski, G., Moderski, R., \& Poutanen, J. 1997, ApJ, 484, 108

Stacy, J. G., et al. 1996, A\&AS, 120, 549

Stark, A. A., et al. 1992, ApJS, 79, 77

Stocke, J. T., et al. 1985, ApJ, 298, 619

Takahashi, T., et al. 1996, ApJ, 470, L89

Tanaka, Y., Inoue, H., \& Holt, S. S. 1994, PASJ, 46, L37

Tashiro, M. 1992, Ph. D. Thesis, University of Tokyo

Tashiro, M., et al. 1995, PASJ, 47, 131 
Thompson, D. J., et al. 1995, ApJS, 101, 259

Ulrich, M.-H., Maraschi. L., \& Urry, C. M. 1997, ARA\&A, 35, 445

Urry, C. M., \& Padovani, P. 1995, PASP, 107, 803

Urry, C. M., et al. 1996, ApJ, 463, 424

Urry, C. M., et al. 1997, ApJ, 486, 799

Vermeulen, R. C., \& Cohen, M. H. 1994, ApJ, 430, 467

Veron-Cetty, M.-P., \& Veron, P. 1993, ESO Scientific Report 13

Vestrand, W. T., Stacy, J. G., \& Sreekumar, P. 1995, ApJ, 454, L93

von Montigny, C., et al. 1993, A\&AS, 97, 101

von Montigny, C., et al. 1995, ApJ, 440, 525

von Montigny, C., et al. 1997, ApJ, 483, 161

Xie, G. Z., et al. 1992, ApJS, 80, 683

Yamashita, A., et al. 1997, IEEE Trans. Nucl. Sci, 44, 847 
Table 1. ASCA observation of blazars.

\begin{tabular}{|c|c|c|c|c|c|c|}
\hline source & other name & redshift & $N_{\mathrm{H}}^{\text {Gal a }}$ & date $^{b}$ & $\alpha_{X}^{\mathrm{c}}$ & flux ${ }^{\mathrm{d}}$ \\
\hline \multicolumn{7}{|c|}{ HBL } \\
\hline $0323+022$ & $\mathrm{H}$ & 0.147 & 8.68 & $94 \operatorname{Jan} 24$ & $1.74_{-0.13}^{+0.19}$ & $(1.0 \pm 0.1) \times 10^{-12}$ \\
\hline $0414+009$ & $\mathrm{H}$ & 0.287 & 9.15 & 96 Aug 30 & $1.62_{-0.05}^{+0.04}$ & $(7.4 \pm 0.1) \times 10^{-12}$ \\
\hline $0548-322$ & PKS & 0.069 & 2.49 & 93 Oct 30 & $0.96_{-0.02}^{+0.02}$ & $(3.4 \pm 0.1) \times 10^{-11}$ \\
\hline $1101+384$ & Mkn 421 & 0.031 & 1.45 & 93 May 10 & $1.97_{-0.03}^{+0.02}$ & $(2.4 \pm 0.1) \times 10^{-11}$ \\
\hline $1426+428$ & $\mathrm{H}$ & 0.129 & 1.37 & 94 Feb 6 & $1.17_{-0.03}^{+0.04}$ & $(1.8 \pm 0.1) \times 10^{-11}$ \\
\hline $1652+398$ & Mkn 501 & 0.034 & 1.73 & *96 Mar 26, 27 & $\begin{array}{l}1.22_{-0.02}^{+0.02} \\
-\end{array}$ & $(6.6 \pm 0.1) \times 10^{-11}$ \\
\hline $2155-304$ & PKS & 0.116 & 1.77 & 94 May 19 & $1.62_{-0.01}^{+0.01}$ & $(7.2 \pm 0.1) \times 10^{-11}$ \\
\hline \multicolumn{7}{|c|}{ LBL } \\
\hline $0235+164$ & $\mathrm{AO}$ & 0.940 & 7.60 & *94 Feb 4,11,16,19 & $0.82_{-0.12}^{+0.14}$ & $(1.4 \pm 0.1) \times 10^{-12}$ \\
\hline $0716+714$ & & $>0.3$ & 3.73 & ${ }^{*} 94 \operatorname{Mar} 16,19,21$ & $1.07_{-0.14}^{+0.10}$ & $(1.3 \pm 0.1) \times 10^{-12}$ \\
\hline $0735+178$ & PKS & $>0.424$ & 4.35 & 96 Apr 22 & $0.76_{-0.23}^{+0.18}$ & $(7.8 \pm 0.7) \times 10^{-13}$ \\
\hline $0851+202$ & OJ287 & 0.306 & 2.75 & 94 Nov 18 & $0.62_{-0.06}^{+0.05}$ & $(5.1 \pm 0.1) \times 10^{-12}$ \\
\hline \multicolumn{7}{|c|}{ QHB } \\
\hline $0208-512$ & PKS & 1.003 & 3.17 & *95 Jan 15 & $0.66_{-0.09}^{+0.10}$ & $(7.9 \pm 0.3) \times 10^{-12}$ \\
\hline $0333+321$ & NRAO140 & 1.258 & 14.22 & $94 \mathrm{Feb} 1$ & $0.60_{-0.05}^{+0.04}$ & $(9.7 \pm 0.2) \times 10^{-12}$ \\
\hline $0528+134$ & PKS & 2.060 & 25.30 & *95 Mar 7,14,19 & $0.58_{-0.04}^{+0.05}$ & $(1.1 \pm 0.1) \times 10^{-11}$ \\
\hline $1226+023$ & $3 \mathrm{C} 273$ & 0.158 & 1.81 & *93 Dec 20,23,27 & $0.51_{-0.01}^{+0.01}$ & $(1.7 \pm 0.1) \times 10^{-10}$ \\
\hline $1252-055$ & 3C 279 & 0.538 & 2.22 & *93 Dec $21,23,27$ & $0.65_{-0.07}^{+0.06}$ & $(1.0 \pm 0.1) \times 10^{-11}$ \\
\hline $1633+382$ & $4 \mathrm{C} 38.41$ & 1.814 & 1.00 & *96 Mar 21,25,27 & $0.51_{-0.31}^{+0.32}$ & $(2.1 \pm 0.3) \times 10^{-12}$ \\
\hline $2230+114$ & CTA 102 & 1.037 & 5.05 & *95 Dec 6 & $0.58_{-0.12}^{+0.12}$ & $(3.1 \pm 0.2) \times 10^{-12}$ \\
\hline
\end{tabular}

${ }^{a}$ Galactic column density in the units of $10^{20} \mathrm{~cm}^{-2}$. Elvis, Wilkes, \& Lockman (1989) when available, Stark et al. (1992) otherwise. For PKS 0208-512 the value is from Dickey \& Lockman (1990).

$\mathrm{b}_{*}$ symbol show simultaneous observation with ASCA and EGRET.

${ }^{\mathrm{c}} 2-10 \mathrm{keV}$ energy index obtained with the absorption fixed at Galactic value.

${ }^{\mathrm{d}}$ Absorbed $2-10 \mathrm{keV}$ flux in unit of $\mathrm{erg} \mathrm{s}^{-1} \mathrm{~cm}^{-2}$. Systematic errors $10 \%$ are not included.

Errors are quoted at $90 \%$ confidence limit for single parameter. 
Table 2. Results of multiband analysis.

\begin{tabular}{|c|c|c|c|c|c|c|c|c|c|c|}
\hline \multirow[t]{2}{*}{ source } & \multicolumn{3}{|c|}{$\log$ frequency $(\mathrm{Hz})^{\mathrm{a}}$} & \multicolumn{3}{|c|}{$\log \operatorname{luminosity}\left(\operatorname{erg~s}^{-1}\right)^{\mathrm{b}}$} & \multicolumn{3}{|c|}{$\Delta t$} & \multirow[t]{2}{*}{$\delta^{\mathrm{c}}$} \\
\hline & $\nu_{L E}{ }^{\mathrm{d}}$ & $\nu_{H E}$ & $\nu_{S S C}$ & $L_{L E}{ }^{\mathrm{d}}$ & $L_{H E}{ }^{\mathrm{d}}$ & $L_{S S C}$ & (days) & band & ref & \\
\hline $\mathrm{H} 0323+022$ & 15.5 & $\cdots$ & $\ldots$ & 44.4 & $<44.6$ & $<44.6$ & $\cdots$ & $\ldots$ & $\cdots$ & $\cdots$ \\
\hline H0414+009 & 16.8 & $\cdots$ & $\cdots$ & 45.3 & $<45.4$ & $<45.4$ & $\cdots$ & $\cdots$ & $\cdots$ & $\cdots$ \\
\hline PKS0548-322 & 16.8 & $\ldots$ & $\ldots$ & 44.4 & $<44.1$ & $<44.1$ & $\ldots$ & $\ldots$ & $\ldots$ & $\ldots$ \\
\hline Mkn 421 & 16.0 & $24.9 \pm 0.3$ & $24.9 \pm 0.3$ & 44.3 & 43.9 & $43.9 \pm 0.1$ & 0.5 & $\mathrm{X}$ & 1 & 3 \\
\hline $\mathrm{H} 1426+428$ & 17.0 & $\ldots$ & $\ldots$ & 44.7 & $<44.6$ & $<44.6$ & $\ldots$ & $\ldots$ & $\ldots$ & $\ldots$ \\
\hline Mkn 501 & 16.3 & $24.2 \pm 0.6$ & $24.2 \pm 0.6$ & 44.2 & 44.3 & $44.3 \pm 0.4$ & 1 & $\gamma$ & 2 & 1 \\
\hline PKS2155-304 & 16.2 & $>23.9$ & $>23.9$ & 45.8 & $>45.2$ & $>45.2$ & 0.5 & $\mathrm{X}$ & 3 & 2 \\
\hline $\mathrm{AO} 0235+164$ & 13.6 & $>24.2$ & $\ldots$ & 46.9 & $>46.9$ & $46.9^{\mathrm{e}}$ & 3 & $\mathrm{X}$ & 4 & $6.6 \times 10^{2}$ \\
\hline $0716+714$ & 14.6 & $>23.5$ & $\ldots$ & 45.8 & $>45.8$ & $>45.8$ & 2 & $\mathrm{X}$ & 5 & 18 \\
\hline PKS0735+178 & 14.4 & $<22.3$ & $\ldots$ & 46.3 & $>46.6$ & $>46.6$ & 1 & opt & 6 & 12 \\
\hline OJ287 & 13.9 & $\ldots$ & $\ldots$ & 46.2 & $>46.8$ & $\ldots$ & $\ldots$ & $\ldots$ & $\ldots$ & $\ldots$ \\
\hline PKS0208-512 & 13.6 & $21.0 \pm 0.1$ & $20.6_{-0.7}^{+1.0}$ & 46.9 & 48.4 & $46.5_{-0.4}^{+0.6}$ & 7 & $\gamma$ & 7 & 5 \\
\hline NRAO140 & 13.7 & $\ldots$ & $\ldots$ & 46.2 & $<46.8$ & $<46.8$ & $\ldots$ & $\ldots$ & $\ldots$ & $\ldots$ \\
\hline PKS0528+134 & 13.0 & $22.3 \pm 0.2$ & $18.7_{-0.1}^{+0.7}$ & 47.1 & 48.4 & $46.8_{-0.1}^{+0.4}$ & 2 & $\gamma$ & 8 & $2.5 \times 10^{2}$ \\
\hline $3 \mathrm{C} 273$ & 13.5 & $20.2 \pm 0.1$ & $20.2 \pm 0.1$ & 45.8 & 46.2 & $46.2 \pm 0.1$ & 1 & opt & 9 & 5 \\
\hline $3 \mathrm{C} 279$ & 13.7 & $23.6 \pm 0.4$ & $20.7_{-0.7}^{+0.9}$ & 46.7 & 47.5 & $46.6_{-0.3}^{+0.5}$ & 2 & $\gamma$ & 10 & $1.5 \times 10^{2}$ \\
\hline $4 \mathrm{C} 38.41$ & 13.9 & $23.7 \pm 0.2$ & $21.1_{-0.9}^{+1.1}$ & 46.4 & 48.2 & $46.7_{-0.6}^{+1.3}$ & 2 & $\gamma$ & 11 & 86 \\
\hline CTA 102 & 13.5 & $21.6 \pm 0.1$ & $20.1_{-0.8}^{+0.9}$ & 46.4 & 47.9 & $46.1_{-0.4}^{+0.6}$ & 2 & opt & 12 & 21 \\
\hline
\end{tabular}

${ }^{\mathrm{a}}$ rest-frame peak frequency

bobserver-frame luminosity at the peak frequency assuming $4 \pi$ radiation

${ }^{\mathrm{c}}$ lower limit assuming $L_{H E}=L_{S S C}$; see the text

derror is \pm 0.1

eassuming $L_{S S C}=L_{L E}$; see the text

References. - (1) Takahashi et al. 1996; (2) Aharonian et al. 1997; (3) Kubo 1997, Urry et al. 1997; (4)Madejski et al. 1996; (5) Cappi et al. 1994; (6) Xie et al. 1992; (7) Bertsch et al. 1993; (8) Hunter et al. 1993b; (9)Courvoisier et al. 1988; (10) Kniffen et al. 1993; (11) Mattox et al. 1993; (12) Pica et al. 1988 


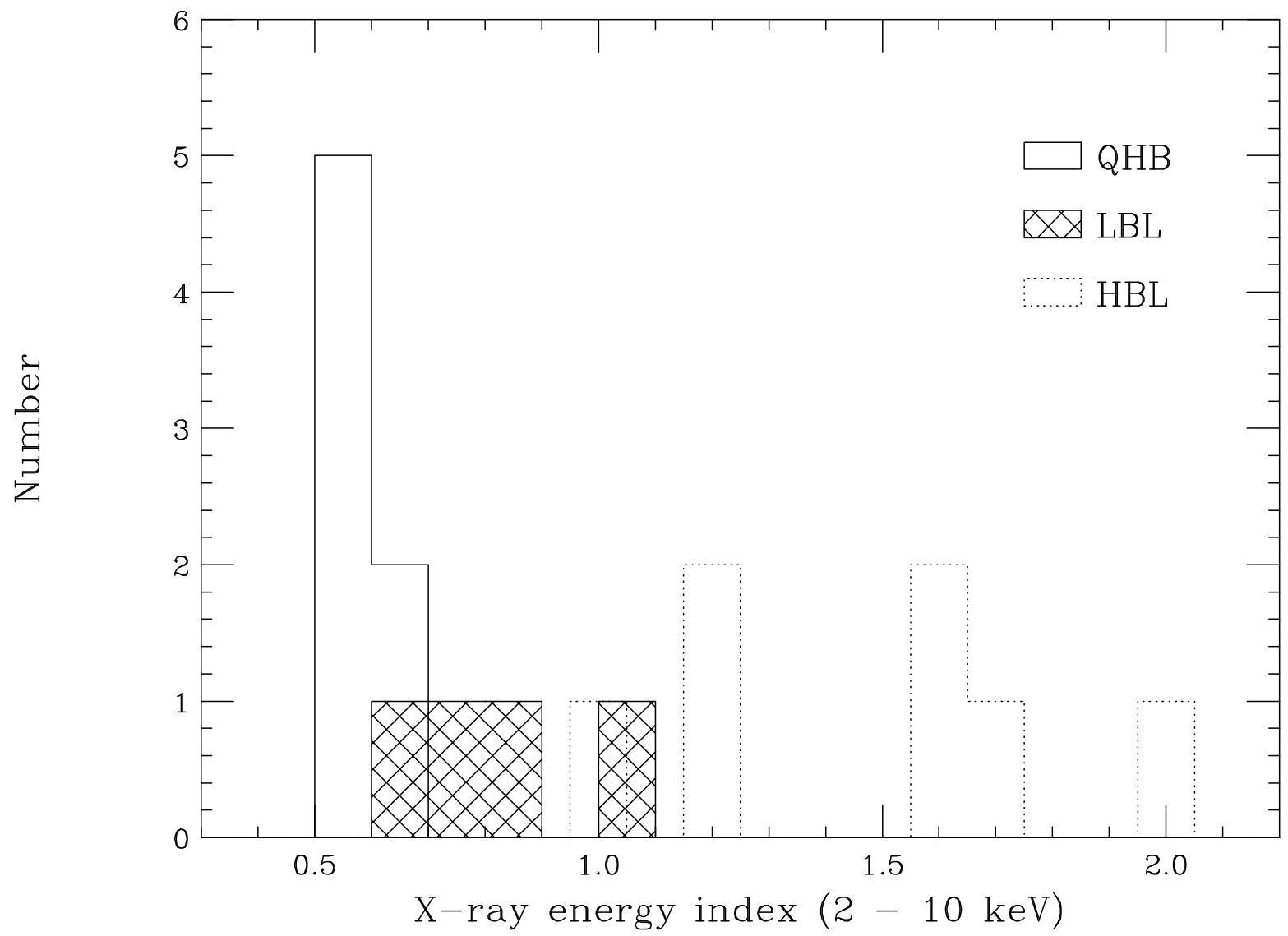

Fig. 1.- Distribution of power law energy indices observed by $A S C A$ in the $2-10 \mathrm{keV}$ in the observer frame. The spectra were assumed to be power laws with Galactic absorption. 

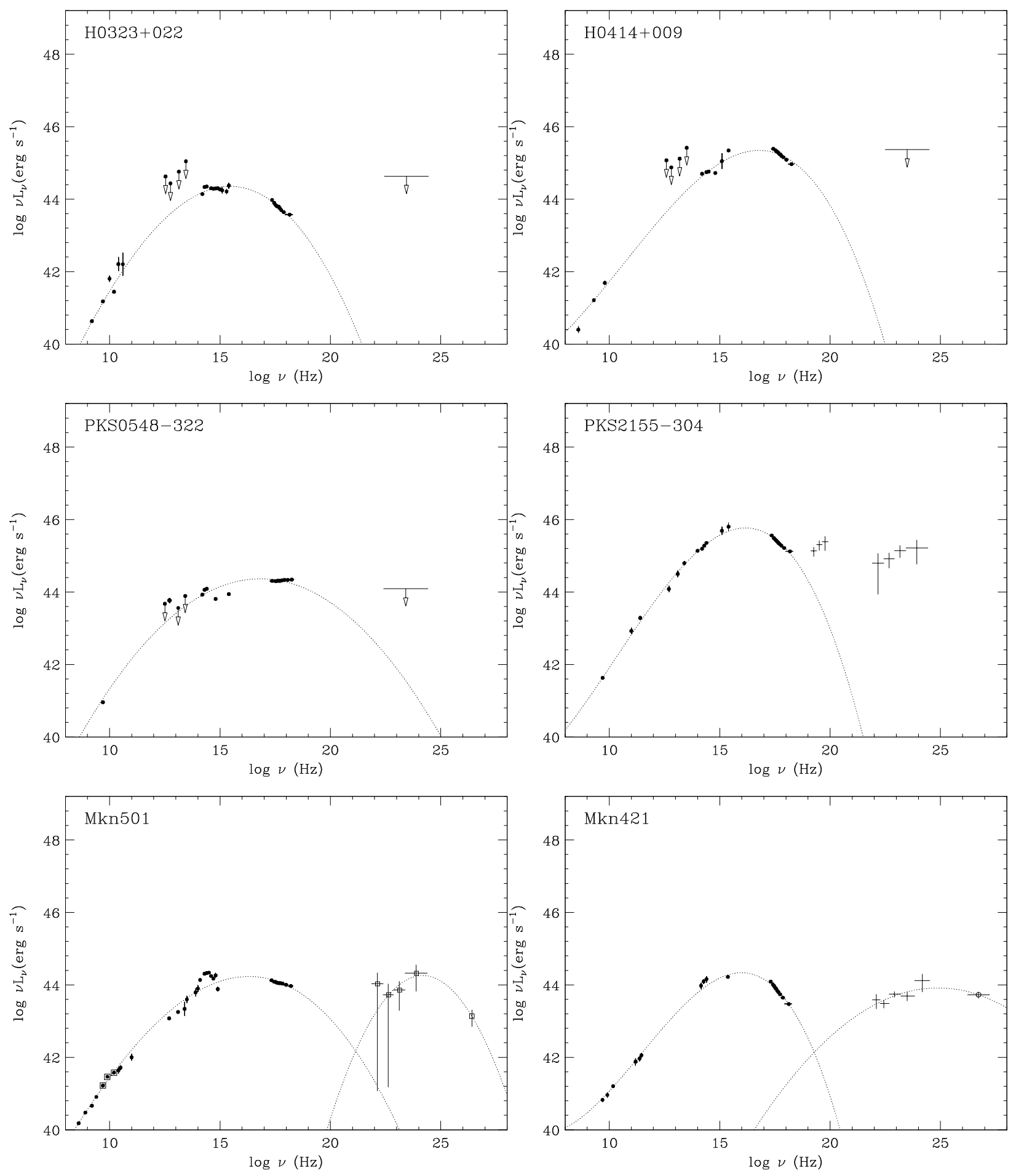

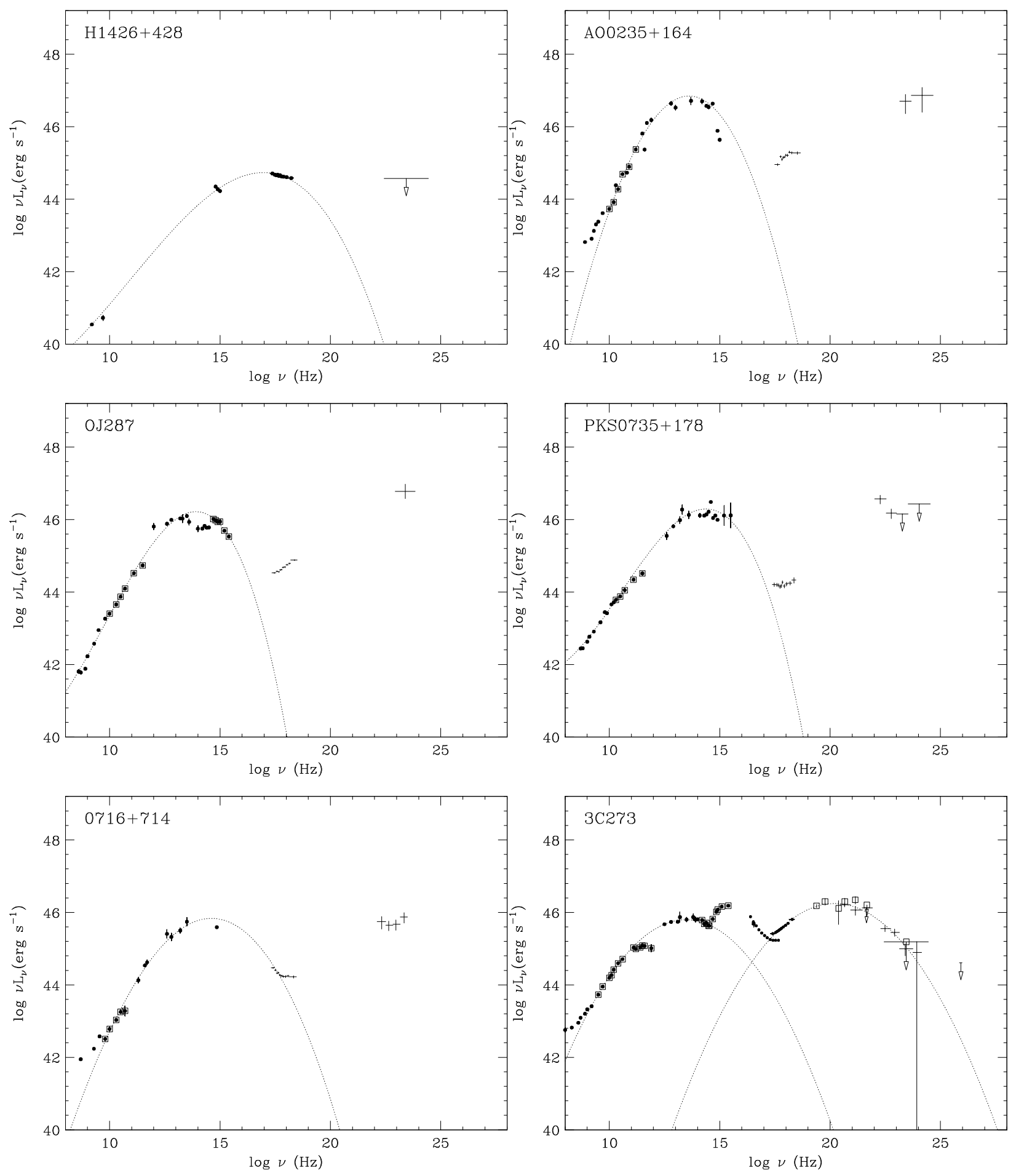

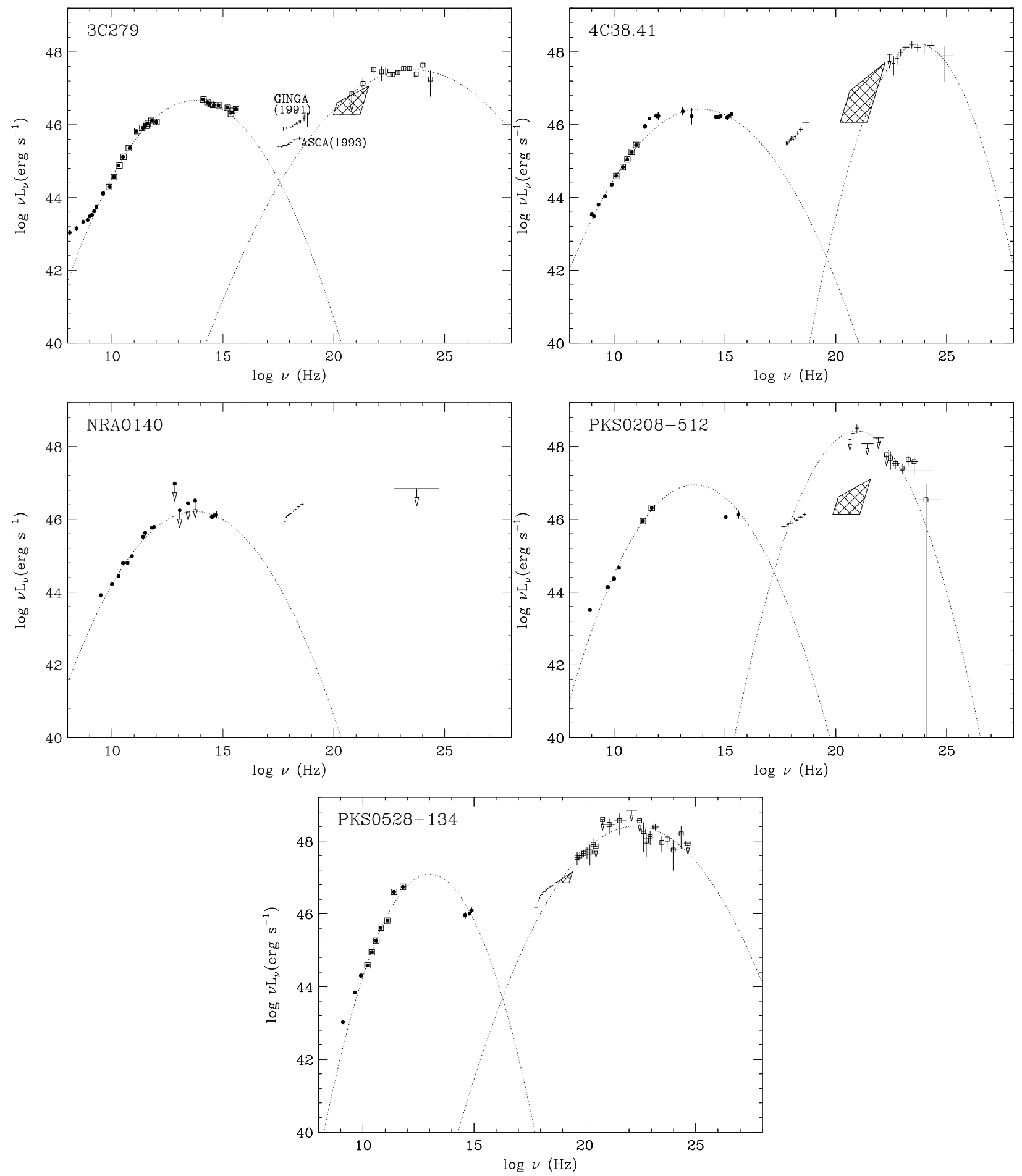

Fig. 2.- Multiband spectra of blazars, showing the distribution of flux versus the restframe frequency. Square symbols show simultaneous observations with ASCA, although for radio data they include quasi-simultaneous observations. The dashed line is the polynomial 
fit to the spectrum. The X-ray spectra are corrected for absorption using the Galactic column density. Hatched region in QHBs corresponds to the estimated range of $\nu_{S S C}$ and $L_{S S C}$. For 3C 279, we plot the ASCA as well as the Ginga X-ray spectra; for this object, the squares represent observations contemporaneous with the plotted Ginga data (see also Maraschi et al. 1994b). The data from the radio to optical bands were mainly taken from NED database. The radio data were also taken from UMRAO database, and Teräsranta \& Tornikoski in private communication. We also used data from Bersanelli et al. 1992 (optical), Pian \& Treves 1993 (UV), Falomo et al. 1994 (optical), Giommi, Ansari \& Micol 1995 (radio to X-ray), Impey et al. 1988 (IR), Neugebauer et al. 1986 (IR), McNaron-Brown et al. 1995 (OSSE, COMPTEL, EGRET), Bloom et al. 1994 (radio, mm, IR), Fichtel et al. 1994 (EGRET upper limit). Additional data for each object were taken from H0323+022 (Feigelson et al. 1986; Jannuzi et al. 1993; Falomo et al. 1993), H0414+009 (McHardy et al. 1992), PKS 2155-304 (Vestrand, Stacy, \& Sreekumar 1995), Mkn 421 (Macomb et al. 1995), Mkn 501 (Kataoka et al. 1998), H1426+428 (Remillard et al. 1989), OJ287 (Landau 1983; Idesawa et al. 1997; Shrader, Hartman, \& Webb 1996), AO0235+164 (Madejski et al. 1996), PKS 0735+178 (Nolan et al. 1996), 0716+714 (Lin et al. 1995), 3C 273 (von Montigny et al. 1993; von Montigny et al. 1997; ROSAT : MPE Annual Report 1993), 3C 279 (Hartman et al. 1996), PKS 0208-512 (Veron-Cetty \& Veron 1993; Blom et al. 1995; Stacy et al. 1996), PKS 0528+134 (Rieke et al. 1982; Collmar et al. 1998), 4C 38.41 (Landau 1986; Mattox et al. 1993) 


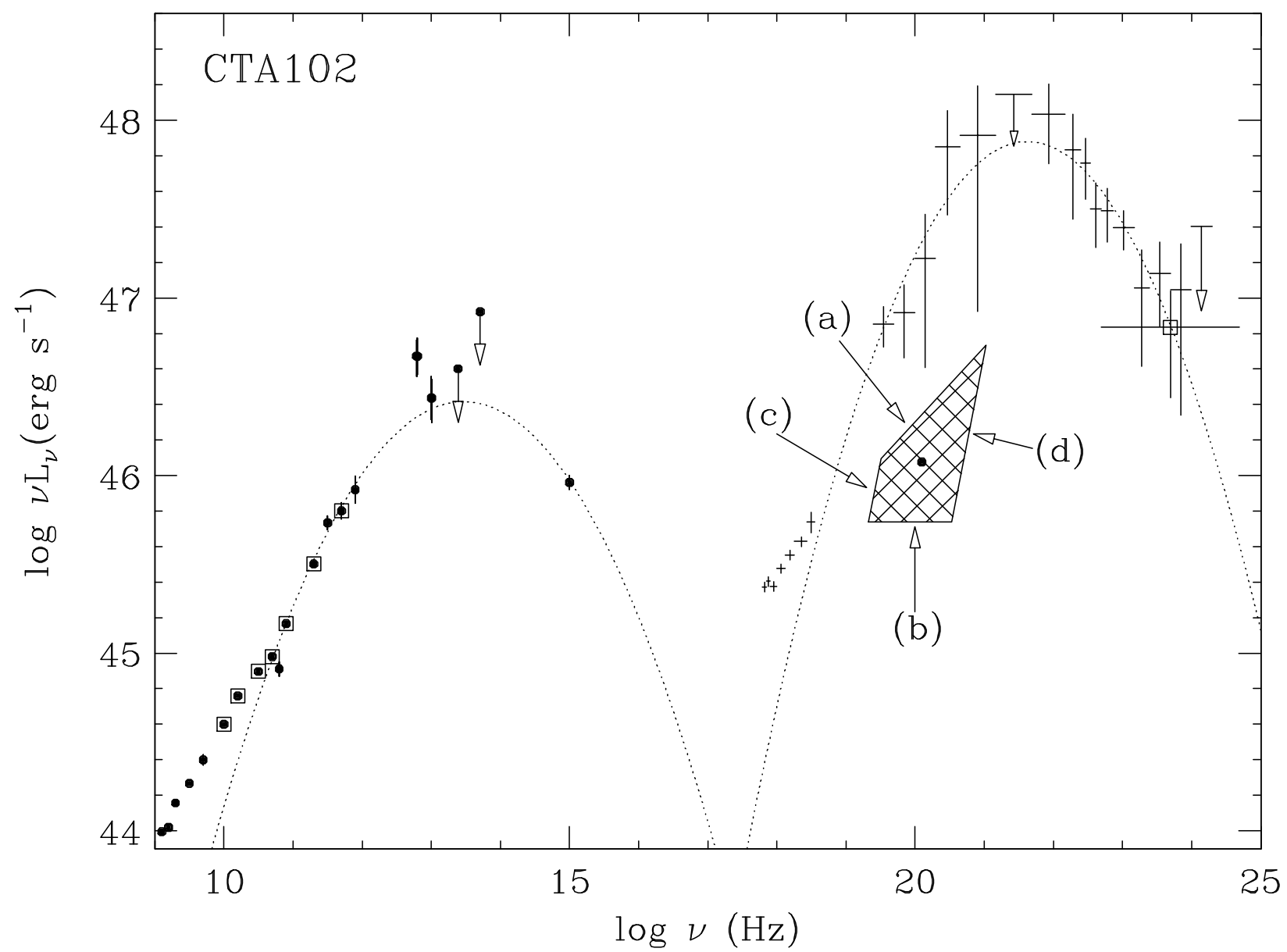

Fig. 3.- Multiband spectrum of CTA 102, where we illustrate in more detail the procedure for choosing input parameters for the model used in the text. Hatched region corresponds to the estimated range of $\nu_{S S C}$ and $L_{S S C}$. The lines bounding the shaded area are: (a) a linear extrapolation of the ASCA spectrum, (b) the highest value of $\nu F(\nu)$ measured by $A S C A$, (c) $\left(\nu_{S S C}, L_{S S C}\right)$ for $\delta=5$, and (d) $\left(\nu_{S S C}, L_{S S C}\right)$ for $\delta=20$. The filled symbol in the hatched region shows the point that is used in the calculation of magnetic field $B$ and electron Lorentz factor $\gamma_{b}$. The data used are from sources as given in Fig. 2; in addition, we used Nolan et al. (1993) and Padovani (1992). 


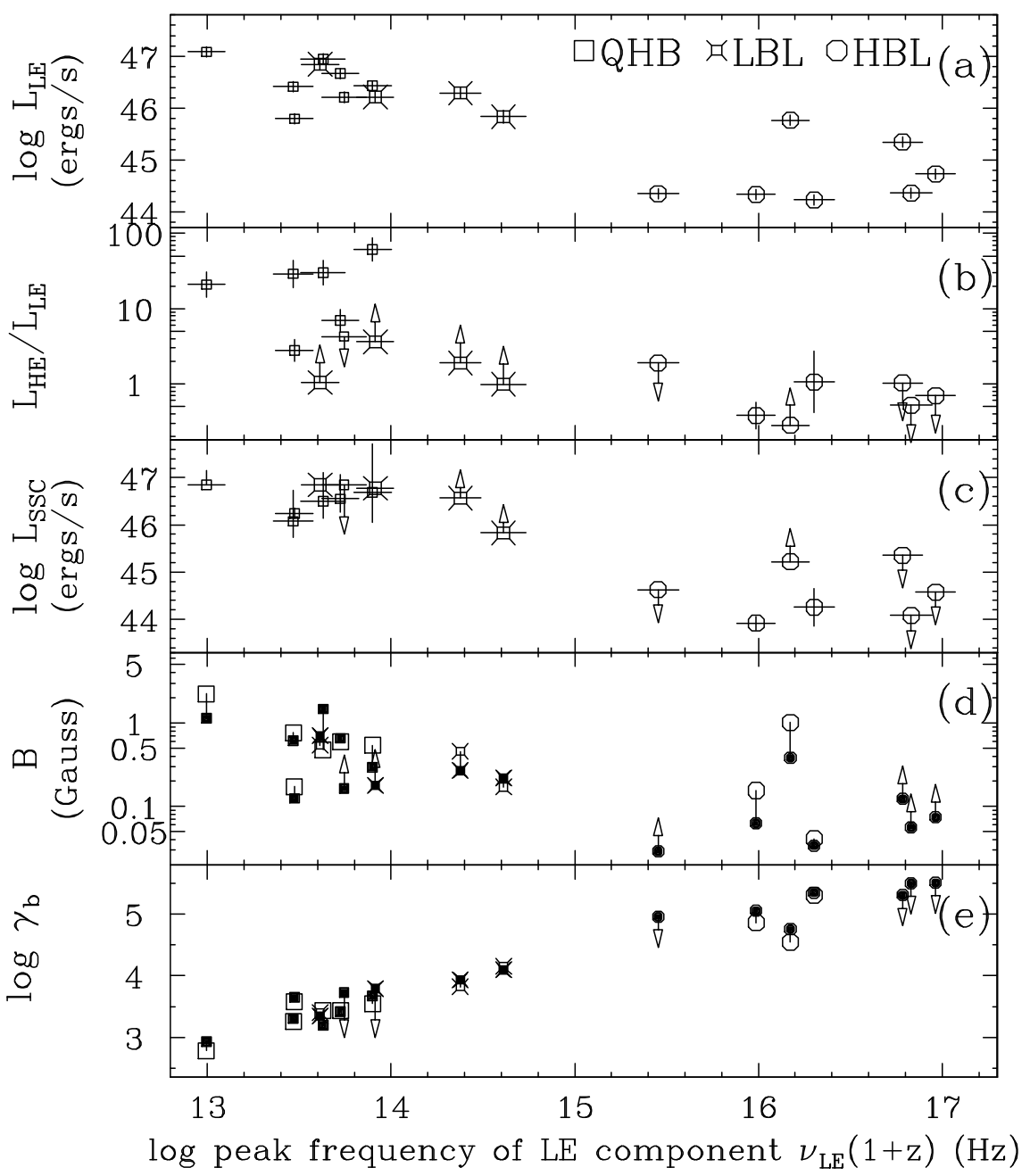

Fig. 4.- The distribution of parameters derived from the multi-band analysis as a function of the rest-frame peak frequency of the LE component. (a) LE luminosity in the observer frame $\left(L_{L E}\right)$; (b) the ratio $L_{H E} / L_{L E}$ (c) SSC luminosity in the observer frame $\left(L_{S S C}\right)$; (d) magnetic field $(B)$; and (e) Lorentz factor of electrons radiating at the peak of the $\nu F(\nu)$ spectrum $\left(\gamma_{b}\right)$. In the calculation, we use the beaming factor $\delta=10$. The size $R$ is estimated from the observed time variability from Table 2 (open symbol). We also plot values calculated with $R=0.01 \mathrm{pc}$ (filled symbol). For HBLs, QHB 3C 273, and two LBLs PKS 0735+178, $0716+714$, we use the $L_{H E}$ as $L_{S S C}$. For LBL AO0235+164 we assume $L_{S S C}=L_{L E}$. For QHBs except 3C 273, $L_{S S C}$ is estimated by extrapolating the ASCA spectrum. Downward arrows in (b),(c),(e) and upward ones in (d) show sources that are not detected in the $\gamma$-ray band. Upward arrows in (b),(c),(e) and downward ones in (d) show sources whose $\gamma$-ray emission is detected, but the peak of the HE component is not determined. 